\title{
Treating sacroiliac joint dislocation through percutaneous sacroiliac screw fixation with the aid of 2 fluoroscopes: a novel technique
}

\author{
Chuangang Peng ${ }^{1 \#}$, Baoming Yuan ${ }^{1 \#}$, Jincheng Wang ${ }^{1,2}$, He Liu ${ }^{1,2}$, Dongsheng Wang ${ }^{1}$ \\ ${ }^{1}$ Department of Orthopedics, The Second Hospital of Jilin University, Changchun, China; ${ }^{2}$ Jilin Clinical Research Center for Orthopaedic Disease, \\ Changchun, China
}

\#These authors contributed equally to this work.

Correspondence to: Dongsheng Wang, MD, PhD. Department of Orthopedics, The Second Hospital of Jilin University, Changchun 130041, China. Email: dongshengwang@163.com.

Background: Percutaneous sacroiliac screw fixation is the standard treatment for sacroiliac joint (SIJ) dislocation. In most hospitals, the procedure is guided by a C-arm X-ray fluoroscopy system, which must be repeatedly repositioned during surgery. In this study, we investigated the feasibility of using 2 fluoroscopes simultaneously.

Methods: A total of 28 consecutive patients with SIJ dislocation were included in this study. The patients were randomly allocated to groups and underwent percutaneous sacroiliac screw fixation using either 1 or 2 fluoroscopes. Total radiation exposure frequency, radiation dose, and operation time were recorded and compared. Dislocation reduction quality was assessed using the Tornetta and Matta standard, and the Majeed functional score was used to evaluate clinical, imaging, and social function following pelvic injury. Complications were also recorded.

Results: The results showed that the radiation exposure frequency was significantly less with 2 fluoroscopes than with a single fluoroscope $(21.5 \pm 8.6$ and $42.6 \pm 18.3$ times, respectively; $\mathrm{P}<0.001)$. However, the radiation dose $(156.3 \pm 67.2 \mathrm{mGy}$ for 1 fluoroscope and $157.8 \pm 38.2 \mathrm{mGy}$ for 2 fluoroscopes; $\mathrm{P}>0.05)$ between the 2 groups was not significantly different. The total operation time was also significantly shorter with 2 fluoroscopic devices than with a single device $(35.8 \pm 12.9$ and $65.5 \pm 19.7$ minutes, respectively; $\mathrm{P}<0.001)$. The dislocation reduction quality and Majeed functional score (92.3\% and $86.7 \%$ for 1 fluoroscope, $93.3 \%$ and $84.6 \%$ for 2 fluoroscopes, respectively; P>0.05) did not differ significantly between the 2 groups at the final follow-up. Complications, such as pain, superficial infection, restricted squatting, limp, and screw failure, were rarely recorded in either group.

Conclusions: The simultaneous application of 2 fluoroscopes is highly appropriate during percutaneous sacroiliac screw fixation to treat SIJ dislocation, and can significantly reduce radiation exposure frequency and operation time.

Keywords: Percutaneous sacroiliac screw; sacroiliac joint dislocation (SIJ dislocation); surgical efficiency; two fluoroscopes

Submitted Mar 17, 2020. Accepted for publication Dec 15, 2020.

doi: 10.21037/qims-20-448

View this article at: http://dx.doi.org/10.21037/qims-20-448 


\section{Introduction}

With the development of convenient modern transportation and high-rise buildings, injuries caused by car accidents and falls are becoming increasingly common. Such injuries can affect the sacroiliac joint (SIJ), which is a weightbearing joint in humans (1). If SIJ dislocation is not treated promptly, complications such as lower back pain, hip pain, and unequal lower limb length can occur (2). Traditionally, conservative treatment methods have been used; however, these require patients to remain in bed for extended periods, which is not conducive to recovery (3). When the SIJ dislocation is reduced, the joint is returned to its standard anatomical position as far as possible, which helps to restore the integrity of the pelvis and significantly improves pelvic stability (4).

A variety of surgical procedures can be used to treat SIJ dislocation, including internal fixation with an adjustable plate, tension band plate fixation, and sacral screw fixation (5-8). Biomechanical testing has confirmed that sacral screw fixation is more effective than plate fixation (9). Navigation technology allows for accurate positioning of the screw, minimal trauma and bleeding, a short operation time, a simple operating procedure, and rapid fracture healing (10), making it ideal for guiding SIJ fixation (11). However, most primary hospitals do not have access to computer navigation technology. C-arm X-ray systems are the most commonly used devices to guide fracture reduction, especially in primary hospitals. Hence, investigations to improve the accuracy and effectiveness of the percutaneous sacroiliac screw method, using the guidance of a C-arm X-ray system, is warranted.

The aim of this study was to investigate whether the use of 2 fluoroscopes simultaneously could reduce the radiation exposure frequency, dose and operation time of percutaneous sacroiliac screw fixation in patients with SIJ dislocation. Related clinical outcomes were compared between patients who underwent surgery guided by 2 fluoroscopes and those whose procedures were performed using a single fluoroscope.

\section{Methods}

\section{Inclusion and exclusion criteria}

The inclusion criteria for patients were as follows: (I) aged 18-75 years old; (II) clinically diagnosed with SIJ dislocation; and (III) sacroiliac screw was sufficient to meet the goal of anatomical reduction of the SIJ dislocation.
The patient exclusion criteria were as follows: (I) patients whose physical condition could not tolerate surgery; (II) patients diagnosed with other pelvic injuries; and (III) patients who could not commit to follow-up for longer than 24 months.

This was a prospective randomized trial, which was conducted in accordance with the principles outlined in the Declaration of Helsinki, and it was granted approval by the Ethics Committee of the Second Hospital of Jilin University (No. 20160321). Written informed consent to participate was given by all patients involved in the study. Participant data were anonymized to ensure confidentiality and privacy.

The power for the radiation exposure frequency and radiation dose were calculated using PASS 11 software (NCSS, East Kaysville, UT, USA) based on a 2-sample $t$-test power analysis with a significance level of $5 \%$. With a sample size of 11 participants treated using 1 fluoroscope and 11 participants treated using 2 fluoroscopes, the trial would have more than $92 \%$ power to detect a difference between 2 groups in the proportion of participants with a radiation time greater than $5 \%$. Finally, 28 consecutive patients who sustained SIJ dislocation were included in the study; SIJ dislocation was caused by traffic accidents and falling from a height in 20 and 8 cases, respectively. Preoperative X-ray (Figure 1A) and three-dimensional (3D) computed tomography (CT) examination of the pelvis were performed conventionally. Before surgery, the patients were randomly divided into 2 groups. The study group consisted of patients who were operated on using 2 fluoroscopes, while the control group comprised patients operated with a single fluoroscope. The type of $\mathrm{C}$-arm $\mathrm{X}$-ray machine was Brivo OEC 715, and its single radiation dose was 3.67 mGy. All participants were operated on by a single senior surgeon in the department.

\section{Planned operative technique}

The steps of operations were performed sequentially as follows: (I) with the patient lying supine, the $\mathrm{C}$-arm machine projection angle at the pelvic entrance position, determined from the CT sagittal section (Figure 1B), was positioned at 30-40 degrees to the horizontal line on the ground, unobstructed by the pubic bone; (II) the screw channel on transverse plane CT (Figure 1C) was designed so that screw entry was optimal (the upper edge of the first countersink was $5-12 \mathrm{~mm}$ and the abdominal and head inclination angles were 25 and 10-15 degrees, respectively). 

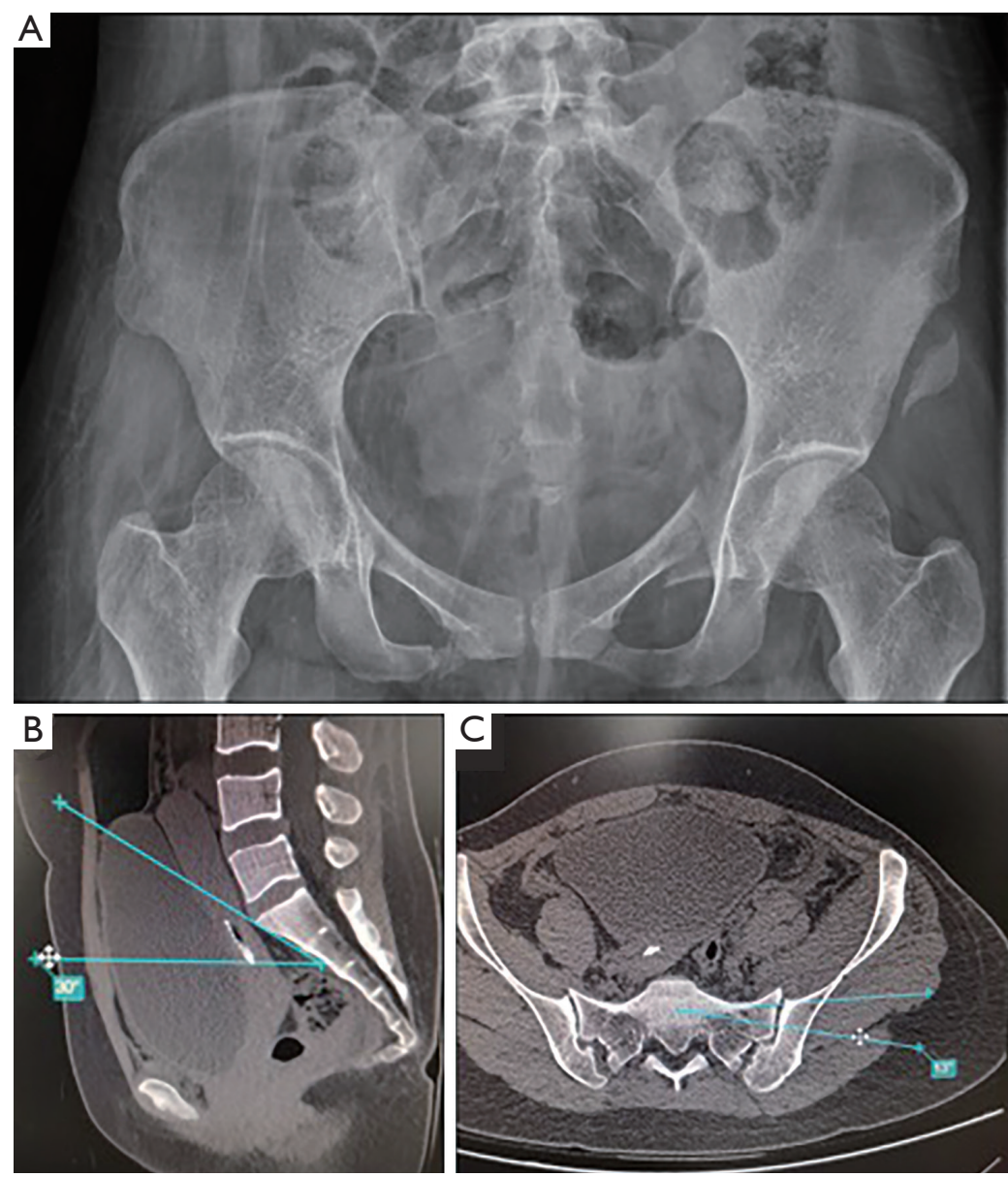

Figure 1 Preoperative X-ray of a patient who sustained left SIJ dislocation (A). C-arm machine projection angle at the pelvic entrance position, determined from the CT sagittal section (B). Screw channel design on transverse plane CT (C). SIJ, sacroiliac joint; CT, computerized tomography.

\section{Positioning of patients and fluoroscopes}

General anesthesia was routinely performed with patients in a supine position with $5 \mathrm{~cm}$ of padding under the buttocks. A C-arm machine was used to visualize the entrance position (Figure $2 A$ ), exit position (Figure $2 B$ ), and the sacrum lateral position (Figure $2 C$ ). The anterior margin of the SIJ and the anterior sacrum were visualized at the entrance position, along with the first sacral foramen on the affected side and the upper edge of the sacral wing near the tangent to the exit position.

The $2 \mathrm{C}$-arm machines were placed on each side of the operating table, according to the previously measured angle (Figure $3 A, B$ ), so that 2 images from different angles could be viewed simultaneously (Figure $3 C$ ) when the 2 pedals were operated together (Figure 3D). This ensured there was no need to move the $\mathrm{C}$-arm machine during surgery; it was only necessary to adjust the position of the guide needle. The operated area was regularly sterilized and draped with sterile sheets, according to standard practice (Figure $3 E$ ). The arc-shaped arm of the machine was wrapped with sterile towels to avoid intraoperative contamination, and the surgeon was able to perform the surgical procedures in the gap beneath the C-arm machine (Figure 3F).

\section{Surgical technique}

A Kirschner wire $(\varnothing=2.5 \mathrm{~mm})$ was pressed against the skin at the potential insertion point, and a camera was used for visualization. When the position of the insertion point was satisfactory, a small incision $(\sim 10 \mathrm{~mm})$ was made in the corresponding skin. The 2 images were used for real-time 

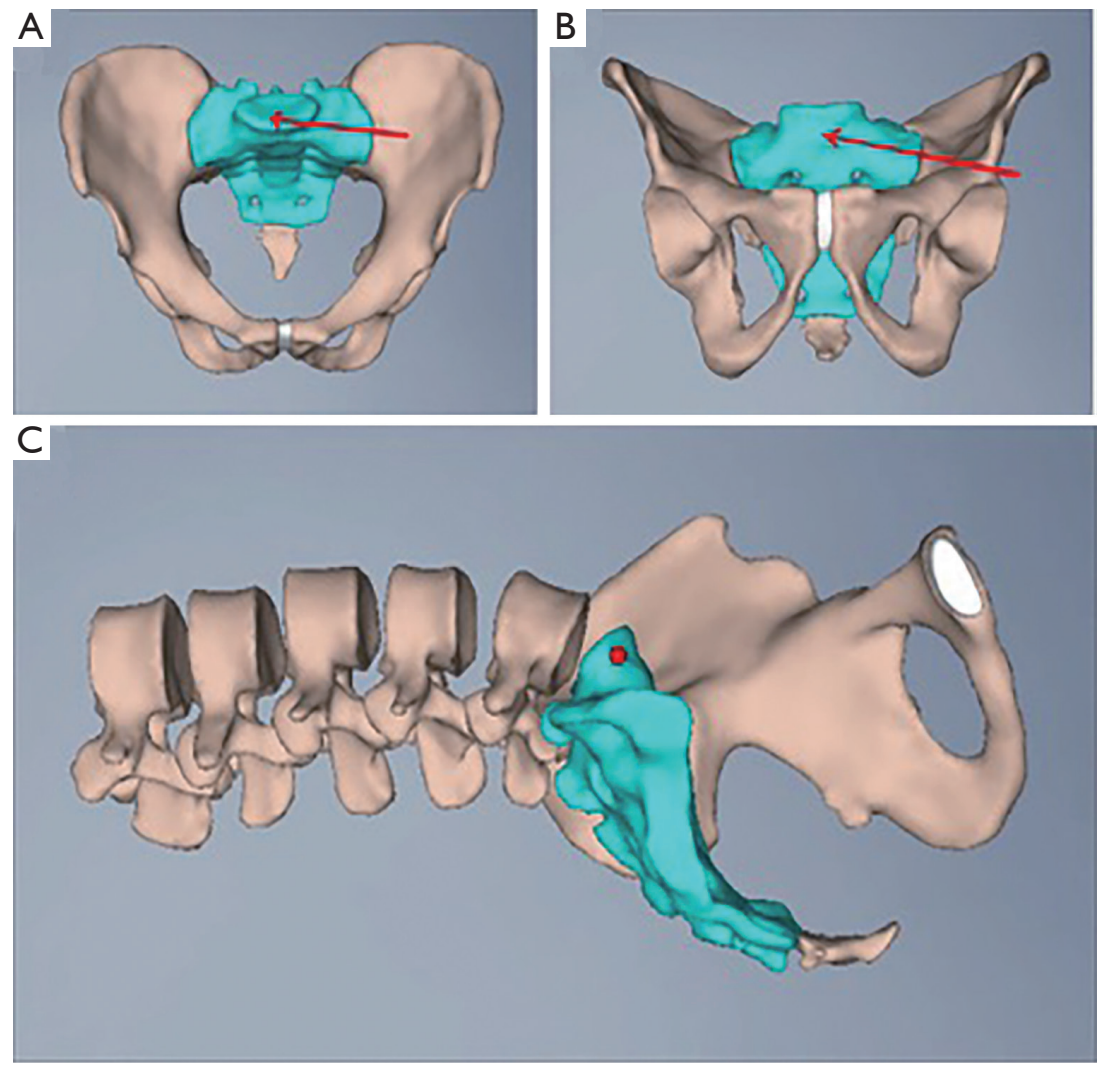

Figure 2 C-arm machine projection angles at the pelvic entrance position (A), exit position (B), and the sacrum lateral position (C), illustrated on a digital model. Red arrows mark the needle direction. The red dot indicating the needle entry point.

monitoring, which allowed the guide needle to be inserted accurately. The position of the guide needle was closely monitored with the help of $2 \mathrm{C}$-arm machines to avoid damage to the presacral venous plexus and sacral nerve (Figure 4A,B,C). A sacroiliac screw was implanted along the guide pin (Figure 4D,E), and then use of the dual C-arm was completed. Finally, the position of the sacroiliac screw was verified again from the lateral view before the incision was closed (Figure 4F).

\section{Postoperative evaluation}

Postoperative evaluation of SIJ dislocation included assessment of fracture reduction, fracture healing, and functional recovery. Fracture reduction and fracture healing were assessed by an independent orthopedic surgeon using the Tornetta and Matta standard (12). Postoperative functional recovery was evaluated using the Majeed functional score to comprehensively assess clinical, imaging, and social function at 6 and 12 months postoperatively, and then annually thereafter (13). Complications, such as pain, superficial infection, restricted squatting (being unable to squat completely), limp, and screw failure were also recorded.

\section{Statistical analysis}

Total radiation exposure frequency was collected from the fluoroscopic devices at the end of the reduction and at the end of surgery, and radiation dose was calculated by multiplying total exposure frequency and single radiation dose by the number of fluoroscopes used. Procedure durations, from incision to wound closure, were collected and compared between the 2 groups. Data were presented as mean \pm standard deviation, and differences in median values between the 2 groups were also assessed. Differences were evaluated using the chi-square test for nominal variables and a 2 -sample 2 -sided independent population 

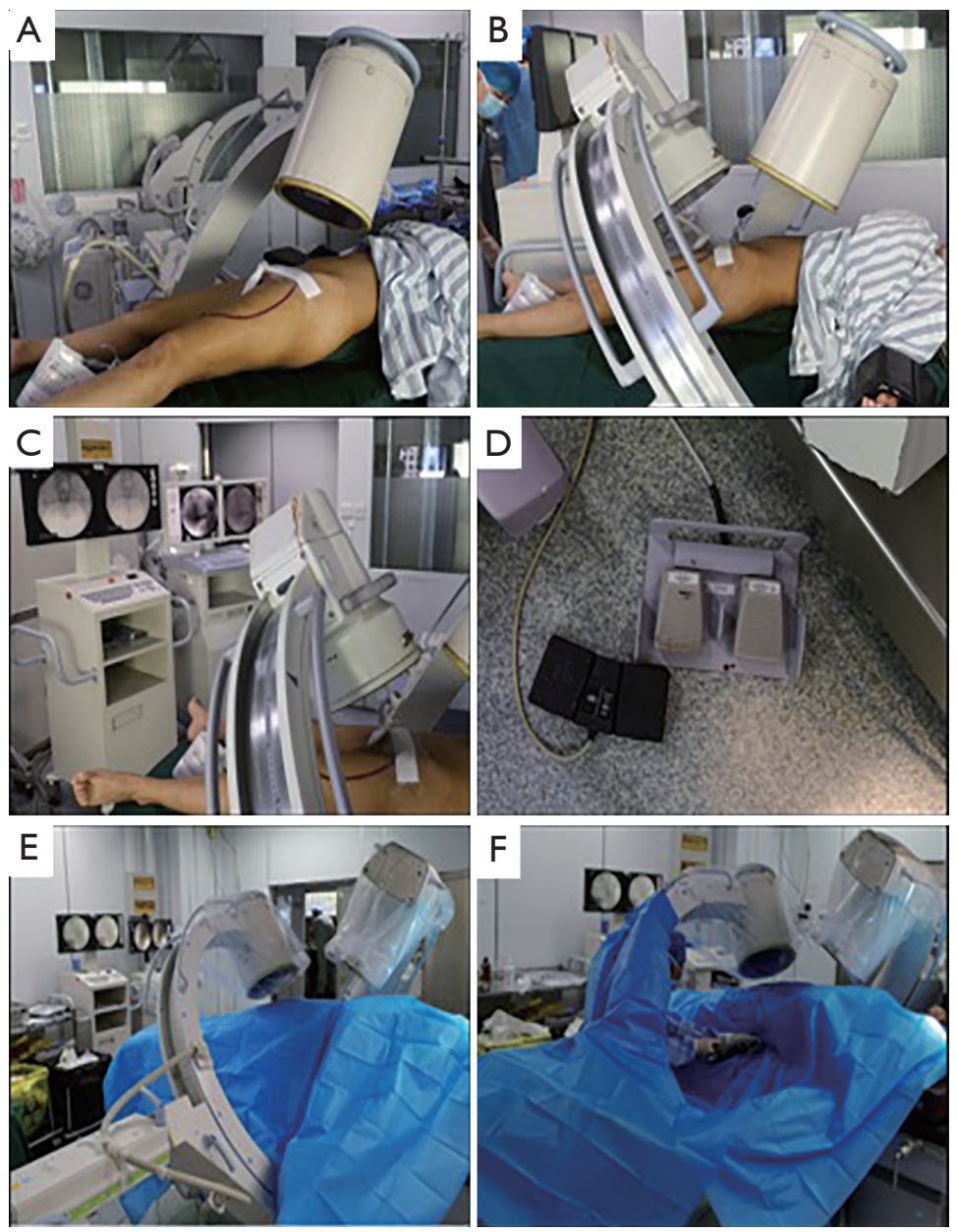

Figure 3 Two C-arm X-ray devices were used to visualize the entrance position (A) and exit position (B) in the operating room. Both images (C) could be obtained simultaneously using 2 pedals placed side by side (D). Draped sterile sheets (E). Photograph showing the position of the surgeon $(\mathrm{F})$.

t-test. A $\mathrm{P}$ value $<0.05$ was considered to be statistically significant.

\section{Results}

\section{Patient information and intra-operative findings}

A total of 28 patients (mean age, 42.6 years; median age, 44 years) participated in the study, including 12 females (42.9\%) and 16 males (57.1\%). The envelope draw method was used to randomly assign 15 patients to receive surgery with the assistance of 1 fluoroscope, and the other 13 to receive surgery with the assistance of 2 fluoroscopes. The total radiation exposure frequency was significantly less in the group operated on with 2 fluoroscopic devices than in the group in which a single device was used $(21.5 \pm 8.6$ and $42.6 \pm 18.3$ times, respectively; $\mathrm{P}<0.001)$. However, there were no significant differences between the 2 groups regarding radiation dose $(156.3 \pm 67.2 \mathrm{mGy}$ for 1 fluoroscope and 157.8 $\pm 38.2 \mathrm{mGy}$ for 2 fluoroscopes; P>0.05). The total operation time was also significantly shorter with 2 fluoroscopic devices than with a single device $(35.8 \pm 12.9$ and $65.5 \pm 19.7$ minutes, respectively; $\mathrm{P}<0.001)$. The results are summarized in Table 1.

\section{Follow-up outcomes}

At the last follow-up, a large majority of patients in both groups demonstrated excellent or good Tornetta and Matta standard values for rates of postoperative fracture 

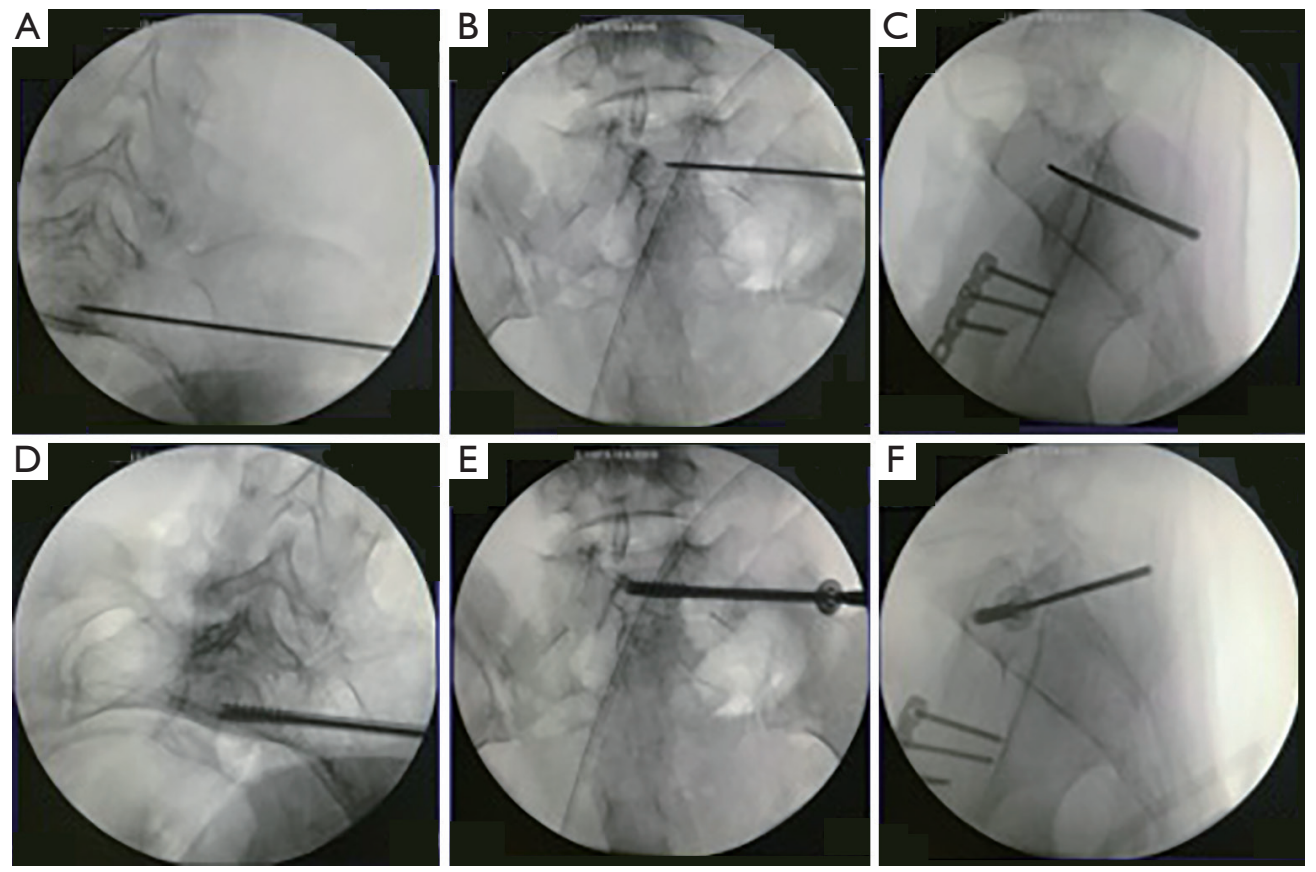

Figure 4 Images of guide needle insertion (A,B,C), sacroiliac screw implantation (D,E), and final verification from the lateral view before closure of the incision $(\mathrm{F})$.

Table 1 Operation time, radiation exposure frequency and radiation dose in patients operated using 1 or 2 fluoroscopes

\begin{tabular}{|c|c|c|c|c|}
\hline Variables & No. of fluoroscopes used & Mean $\pm \mathrm{SD}^{*}$ & Median & $P$ value \\
\hline Operation time (minutes) & 2 fluoroscopes & $35.8 \pm 12.9$ & 38 & \\
\hline \multirow[t]{2}{*}{ Radiation exposure frequency (times) } & 1 fluoroscope & $42.6 \pm 18.3$ & 44 & $<0.001$ \\
\hline & 2 fluoroscopes & $21.5 \pm 5.2$ & 23 & \\
\hline Radiation dose $(\mathrm{mGy})^{\#}$ & 2 fluoroscopes & $157.8 \pm 38.2$ & & \\
\hline
\end{tabular}

*, mean \pm SD presented as mean (standard deviation), " , radiation dose was calculated by multiplying total exposure frequency and single radiation dose by the number of fluoroscopes used.

reduction $(92.3 \%$ and $93.3 \%$ for the groups with 2 and 1 fluoroscope, respectively). Similarly, most patients in both groups had excellent or good Majeed scores for pelvic functional recovery, with mean values of $86.7 \%$ and $84.6 \%$ for the 2 and 1 fluoroscope groups, respectively. Neither the postoperative fracture reduction rate nor pelvic functional recovery differed significantly between the 2 groups $(\mathrm{P}>0.05)$. Moreover, complications, such as pain, superficial infection, restricted squatting, limp, and screw failure, were seldom recorded in either group. Implant removal rates at the final follow-up for the double and single fluoroscope groups were $76.9 \%$ and $80.0 \%$, respectively (Table 2).

\section{Discussion}

Prompt surgery, an appropriate surgical plan, and control of operation time are essential factors influencing the outcomes of patients with SIJ dislocation caused by highimpact injury (14). The sacroiliac screw has become the "gold standard" for treatment of SIJ dislocation, and its biological characteristics and clinical efficacy have been confirmed (15). Behrendt et al. reported that a $3 \mathrm{D}$ computer 
Table 2 Postoperative outcomes and complications

\begin{tabular}{|c|c|c|}
\hline Indices & One fluoroscope & Two fluoroscopes \\
\hline \multicolumn{3}{|c|}{ Radiographic evaluation (Tornetta and Matta standard), n (\%) } \\
\hline Excellent reduction & $11(73.3)$ & $10(76.9)$ \\
\hline Good reduction & $3(20.0)$ & $2(15.4)$ \\
\hline \multicolumn{3}{|l|}{ Functional recovery (Majeed score), n (\%) } \\
\hline Excellent & $9(60.0)$ & $8(61.5)$ \\
\hline Good & $4(26.7)$ & $3(23.1)$ \\
\hline Poor & $2(13.3)$ & $2(15.4)$ \\
\hline Superficial infection & $0(0)$ & $0(0)$ \\
\hline Restricted squatting & $2(13.3)$ & $1(7.7)$ \\
\hline Limp & $2(13.3)$ & $2(15.4)$ \\
\hline Screw failure & $0(0)$ & $0(0)$ \\
\hline Implant removal (according to patient's wishes) & $12(80.0)$ & $10(76.9)$ \\
\hline
\end{tabular}

navigation system could improve the accuracy of sacroiliac screw placement and also prevented operator exposure to the radiation area, although there was no difference in the operation time between surgery conducted using the navigation system and that performed using other methods (10). However, most primary hospitals do not have access to navigation equipment, which restricts its application in these settings. The double-fluoroscopic technique was first reported by Brin et al., who revealed its advantages of easy operation, time efficiency, and good clinical outcomes for closed reduction and internal fixation of stable intertrochanteric fractures (16). Therefore, we attempted to demonstrate that this technique might be also suitable for minimally invasive treatment of SIJ dislocation by percutaneous sacroiliac screw fixation.

The importance of detecting sacral malformations before surgery is well known; such malformations include lumbar sacralization, sacral lumbarization, and congenital malformations of the sacrum. If the slope of the sacral wing is excessively inclined, the screw channel of the SIJ will be extremely narrow, and the anterior cortex will be easily penetrated, which may damage the L5 nerve root and the obturator nerve. Herein, the SIJ screw was preoperatively designed for unilateral fixation of the sacral 1 vertebral body through CT 3D reconstruction. During the operation, 2 C-arm machines were used to dynamically observe the position and direction of the screw, thus significantly reducing the possibility of errors.

We found that using 2 devices could reduce the operation time by $45.3 \%$. This difference in procedure length derives from the fact that, when working with 2 devices, it is not necessary to repeatedly relocate the fluoroscope during the procedure, which is a time-consuming process that is conducted several times when a single fluoroscope is used. Each time the fluoroscope is relocated, the radiology technician and the surgeons have to find the best position, which lengthens the entire procedure. Generally, longer operation times also involve increased tissue exposure time and decreased systemic physiological defenses, which may increase the risk of infection $(17,18)$. Further, shorter procedures can help to accommodate the inclusion of more cases in the operating room schedule.

This study also showed that using 2 fluoroscopes reduced the radiation exposure frequency during surgery by $49.5 \%$ from $42.6 \pm 18.3$ to $21.5 \pm 8.6$ times. The mean addition in radiation exposure frequency for a single operation was 
21.1 times, due to the extra projections required when the fluoroscope was relocated to provide different views during surgery. The surgeon and radiology technician are often dissatisfied with either the image quality or the angle of image capture, requiring the device to be repeatedly readjusted (19). These numerous readjustments lead to increased radiation exposure frequency. When 2 devices are used, there is no need for device relocation for either view, saving time and decreasing radiation exposure frequency. As the patients treated with 2 fluoroscopic devices were exposed to 2 radiation sources simultaneously in a single operation, in theory, their total radiation dose should have been doubled. Hence, there were no significant differences in the radiation dose to surgeons between the groups in our study, even though the radiation exposure frequency with a single fluoroscopic device group was double that with 2 fluoroscopic devices.

Potential disadvantages of the approach described here include that access to $2 \mathrm{C}$-arms for simultaneous use must be feasible for the orthopedic trauma team to implement this technique. Further, surgeons are required to operate in a less comfortable position to avoid contact with the placed machines. Moreover, these findings require replication in a larger cohort to improve confidence in their broad applicability.

\section{Conclusions}

The procedure tested in this study achieved reduced operation times, and protected surgeons and patients from excessive radiation exposure. Therefore, we recommend percutaneous sacroiliac screw fixation using 2 fluoroscopes simultaneously as a feasible alternative option for the treatment of SIJ dislocation.

\section{Acknowledgments}

Funding: This work was supported by the National Natural Science Foundation of China (82001971, 81671804 , and 81772456); Scientific Development Program of Jilin Province (20200802008GH, 20200404202 YY, $20200404190 \mathrm{YY}, 20200404140 \mathrm{YY}, 20200403088 \mathrm{SF}$, $20200802008 \mathrm{GH}, 20200404202 \mathrm{YY}, 20200404140 \mathrm{YY}$, 20190304123 YY, and 20180623050TC); Program of Jilin Provincial Health Department (2019SCZT001, 2019SCZT014, and 2019SRCJ001); and Youth Talents Promotion Project of Jilin Province (192004).

\section{Footnote}

Conflicts of Interest: All authors have completed the ICMJE uniform disclosure form (available at http://dx.doi. org/10.21037/qims-20-448). The authors have no conflicts of interest to declare.

Ethical Statement: The study was conducted in accordance with the Declaration of Helsinki (as revised in 2013). This retrospective study was approved by the local institutional ethical committee of the institutional review board and. Oral and written informed consent was given by all participants, and their associated risks as well as potential complications have been carefully described.

Open Access Statement: This is an Open Access article distributed in accordance with the Creative Commons Attribution-NonCommercial-NoDerivs 4.0 International License (CC BY-NC-ND 4.0), which permits the noncommercial replication and distribution of the article with the strict proviso that no changes or edits are made and the original work is properly cited (including links to both the formal publication through the relevant DOI and the license). See: https://creativecommons.org/licenses/by-nc-nd/4.0/.

\section{References}

1. Day AC, Kinmont C, Bircher MD, Kumar S. Crescent fracture-dislocation of the sacroiliac joint: a functional classification. J Bone Joint Surg Br 2007;89:651-8.

2. Maertens AS, Martin MP 3rd, Dean CS, Rojas D, Parry JA, Maher M, Mauffrey CP. Occult injuries of the contralateral sacroiliac joint in operatively treated pelvis fractures: incidence, root cause analysis, and proposal of treatment algorithm. Int Orthop 2019;43:2399-404.

3. Kokubo Y, Oki H, Sugita D, Takeno K, Miyazaki T, Negoro K, Nakajima H. Functional outcome of patients with unstable pelvic ring fracture. J Orthop Surg (Hong Kong) 2017;25:2309499016684322.

4. Alvis-Miranda HR, Farid-Escorcia H, Alcalá-Cerra G, Castellar-Leones SM, Moscote-Salazar LR. Sacroiliac screw fixation: A mini review of surgical technique. J Craniovertebr Junction Spine 2014;5:110-3.

5. Bai Z, Gao S, Liu J, Liang A, Yu W. Anatomical evidence for the anterior plate fixation of sacroiliac joint. J Orthop Sci 2018;23:132-6.

6. Falzarano G, Rollo G, Bisaccia M, Pace V, Lanzetti RM, Garcia-Prieto E, Pichierri P, Meccariello L. Percutaneous 
screws CT guided to fix sacroiliac joint in tile $\mathrm{C}$ pelvic injury. Outcomes at 5 years of follow-up. Sicot J 2018;4:52.

7. Wu T, Su K, Zhang YZ, Li C, Liu S, Li L. Biomechanical comparison of three kinds of fixation in fracturedislocation of the sacroiliac joint. Technol Health Care 2019;27:89-99.

8. Shui X, Ying X, Mao C, Feng Y, Feng Y, Chen L, Kong J, Guo X, Wang G. Percutaneous screw fixation of crescent fracture-dislocation of the sacroiliac joint. Orthopedics 2015;38:e976-82.

9. Zhang R, Yin Y, Li S, Hou Z, Jin L, Zhang Y. Percutaneous sacroiliac screw versus anterior plating for sacroiliac joint disruption: A retrospective cohort study. Int J Surg 2018;50:11-6.

10. Behrendt D, Mütze M, Steinke H, Koestler M, Josten C, Böhme J. Evaluation of 2D and 3D navigation for iliosacral screw fixation. Int J Comput Assist Radiol Surg 2012;7:249-55.

11. Citak M, Hüfner T, Geerling J, Kfuri M Jr, Gänsslen A, Look V, Kendoff D, Krettek C. Navigated percutaneous pelvic sacroiliac screw fixation: experimental comparison of accuracy between fluoroscopy and Iso-C3D navigation. Comput Aided Surg 2006;11:209-13.

12. Tornetta P 3rd, Matta JM. Outcome of operatively treated unstable posterior pelvic ring disruptions. Clin Orthop Relat Res 1996;329:186-93.

Cite this article as: Peng C, Yuan B, Wang J, Liu H, Wang D. Treating sacroiliac joint dislocation through percutaneous sacroiliac screw fixation with the aid of 2 fluoroscopes: a novel technique. Quant Imaging Med Surg 2021;11(5):2076-2084. doi: 10.21037/qims-20-448
13. Majeed SA. Grading the outcome of pelvic fractures. J Bone Joint Surg Br 1989;71:304-6.

14. Borrelli J Jr, Koval KJ, Helfet DL. The crescent fracture: a posterior fracture dislocation of the sacroiliac joint. J Orthop Trauma 1996;10:165-70.

15. Iorio JA, Jakoi AM, Rehman S. Percutaneous sacroiliac screw fixation of the posterior pelvic ring. Orthop Clin North Am 2015;46:511-21.

16. Brin YS, Palmanovich E, Aliev E, Laver L, Yaacobi E, Nyska M, Kish BJ. Closed reduction and internal fixation for intertrochanteric femoral fractures is safer and more efficient using two fluoroscopes simultaneously. Injury 2014;45:1071-5.

17. Metsemakers WJ, Kuehl R, Moriarty TF, Richards RG, Verhofstad MHJ, Borens O, Kates S, Morgenstern M. Infection after fracture fixation: Current surgical and microbiological concepts. Injury 2018;49:511-22.

18. Ercole FF, Franco LM, Macieira TG, Wenceslau LC, Resende HI, Chianca TC. Risk of surgical site infection in patients undergoing orthopedic surgery. Rev Lat Am Enfermagem 2011;19:1362-8.

19. Kalem M, Başarır K, Kocaoğlu H, Şahin E, Kınık H. The effect of C-arm mobility and field of vision on radiation exposure in the treatment of proximal femoral fractures: A randomized clinical trial. Biomed Res Int 2018;2018:6768272. 